\title{
Recent developments in semiconductor gamma-ray detectors
}

P. N. Luke ${ }^{1}$, M. Amman ${ }^{1}$, C. Tindall ${ }^{1}$, J. S. Lee ${ }^{1}$

${ }^{1}$ Lawrence Berkeley National Laboratory, Berkeley, CA 94720, USA.

\begin{abstract}
The successful development of lithium-drifted Ge detectors in the 1960's marked the beginning of the significant use of semiconductor crystals for direct detection and spectroscopy of gamma rays. In the 1970's, high-purity Ge became available, which enabled the production of complex detectors and multi-detector systems. In the following decades, the technology of semiconductor gamma-ray detectors continued to advance, with significant developments not only in Ge detectors but also in Si detectors and roomtemperature compound-semiconductor detectors. In recent years, our group at Lawrence Berkeley National Laboratory has developed a variety of gamma ray detectors based on these semiconductor materials. Examples include Ge strip detectors, lithium-drifted Si strip detectors, and coplanar-grid CdZnTe detectors. These advances provide new capabilities in the measurement of gamma rays, such as the ability to perform imaging and the realization of highly compact spectroscopy systems.
\end{abstract}




\section{Introduction}

Lithium-drifted Ge (Ge(Li)) detectors were developed and successfully used for gamma-ray spectroscopy in the early 1960s. ${ }^{1}$ The excellent energy resolution and good efficiency achieved by these detectors provided experimenters with unprecedented capabilities in gamma-ray measurements, and this has revolutionized the field of gammaray spectroscopy. In the 1970s, high-purity Ge became available and rapidly replaced $\mathrm{Ge}(\mathrm{Li})$. With high-purity Ge, the detectors are stable at room temperature, and thus the fabrication of detectors can be carried out without the severe time constraints that existed when working with $\mathrm{Ge}(\mathrm{Li})$ detectors. This opened up new possibilities in terms of detector processing, and allowed the production of more diverse types of detectors and multi-detector systems.

In the ensuing decades, there have been continuing advances in semiconductor gamma-ray detector technology. Ge detectors continue to increase in size and complexity. More recently, detectors based on other materials, such as Si and CdZnTe, have also been actively developed for gamma-ray detection. Advances in the technology of semiconductor gamma-ray detectors are the results of contributions from many groups. It is virtually impossible to provide a full account of every development in a conference paper. We will instead describe mainly the recent work carried out at Lawrence Berkeley National Laboratory (LBNL) and focus particularly on device technology. We will reference other developments as appropriate, realizing that omissions are inevitable.

\section{Ge detectors}

By far the most common type of application for Ge detectors is basic gamma-ray spectroscopy, where a single detector is used with a single channel of electronics to provide energy information. With modern low-noise electronics and Ge materials, the best energy resolution achieved with Ge detectors at gamma-ray energies is close to the fundamental limits imposed by charge generation statistics. Therefore, further significant improvement in energy resolution is not expected, unless other non-ionization type detection techniques are used. On the other hand, the efficiency (i.e. volume) of Ge detectors has increased steadily over time. The graph in Fig.1 shows how the maximum volume of high-purity Ge detectors increases with time. The data is compiled from published papers that we believe provide information on the largest high-purity Ge 
detectors available at the time..$^{2-7}$ It is interesting to note that the detector size seems to increase roughly linearly with time. If this trend continues, we will see detectors with volume of 1-liter in a few years!

Besides increased detector volume, more complex detector structures have been developed to provide additional capabilities such as gamma-ray imaging. There are different methods to image gamma rays, including the use of parallel-hole collimators, pinholes, coded apertures, ${ }^{8}$ and Compton cameras. ${ }^{9}$ In almost all cases, position-sensitive detectors are needed to implement an imaging system. A common method to provide position sensitivity is to segment the contacts of a detector. Conventional contacts used in Ge detectors are Li diffused contacts (n-type) and B implanted contacts (p-type). The Li contacts are typically several hundred microns thick, making it difficult to segment using standard etching processes. Often times, mechanical means such as cutting using diamond saws or ultrasonic machining were employed ${ }^{10}$, although segmentation using a photolithography technique has also been developed. ${ }^{11}$ Whatever the segmentation methods employed, the relatively large thickness of the Li contact limits the minimum feature size of contact elements to $>1 \mathrm{~mm}$. In addition, the diffusion of $\mathrm{Li}$, which occurs even at room temperature, could lead to electrical shorts between contact elements when detectors are stored at room temperature for long periods of time, or when radiation damage annealing becomes necessary. Another difficulty in making multi-segment detectors is the instability of the Ge surface. When contacts of a detector are segmented, the intrinsic Ge surface between the contact elements can often become unstable with time or with changes in ambient conditions, which may lead to increased leakage current, increased noise, or inadequate isolation between contact elements. Some type of surface passivation is desirable, but it must be able to be applied to the surface between contacts without affecting the ability to make electrical connections to each contact element.

In recent years, improved contact segmentation techniques have been developed, contributing to the increasing use of segmented Ge coaxial and planar detectors. ${ }^{12-14} \mathrm{At}$ LBNL, we have developed a novel contact technology that simplifies the fabrication of multi-segment detectors, and can potentially increase the yield and reduce the cost of producing such detectors. The contacts we employ make use of an amorphous-Ge (a-Ge) thin film to create an electrically blocking junction between it and the bulk Ge crystal. ${ }^{15}$ The blocking junction permits the application of bias voltage to fully deplete the detector 
while maintaining a low leakage current. The structure of a multi-segment detector using such contacts is shown schematically in Fig. 2. The fabrication process involves the sputter deposition of an a-Ge layer onto the surface of the Ge crystal. Selected areas of the a-Ge film are then metalized to form the contact elements. The resistivity of the a-Ge film is sufficiently high to provide good electrical isolation between contact elements, while the small thickness of the film ensures that it does not affect the detector signals or the passage of signal currents to the metal contacts. An interesting property of the a-Ge contact is its ability to block carrier injection under either polarity of bias voltage. Therefore the same process can be used on both sides of a detector to realize doublesided segmented detectors. One advantage of this contact technology is its simplicity, which involves very few processing steps and can be carried out entirely with the detectors at room temperature. Another advantage is that the a-Ge film covers the full surface of the Ge crystal, forming a passivation layer. There is no need for additional processes to passivate the surface between contact elements afterwards. The amorphous contacts are thin and stable with annealing up to $150{ }^{\circ} \mathrm{C}$. Very small scale contact structures, on the order of $\sim 100$ microns or less, can be realized.

A number of orthogonal-strip detectors have been successfully produced using the a-Ge contact technology. ${ }^{16,17}$ Fig. 3 shows a photograph of a large 64 X 64 orthogonalstrip Ge detector that we have recently fabricated. Practical gamma-ray imaging systems using such detectors are still being actively developed at this time, but early imaging tests have been carried out with prototype systems. Fig. 4 shows images of radioactive sources taken with a Ge orthogonal-strip detector that was fabricated using a-Ge contacts. ${ }^{17} \mathrm{~A}$ pinhole collimator was used to form the image of the sources onto the detector. The image of the three sources can be seen in the upper left of the figure. By setting energy windows at the corresponding gamma-ray lines, the sources can be separately imaged as shown in the other three images in the figure. Because of the excellent energy resolution of the Ge detector, narrow energy windows can be used, and that resulted in the substantial reduction in backgrounds as well. In addition to the two-dimensional position information obtained from the orthogonal strips, the depth of gamma ray interaction in the detector can also be determined by measuring the time difference between signals from strips on opposite sides of the detector. ${ }^{18,19}$ The ability to localize events within the detector in three dimensions improves the accuracy of reconstructed images. 


\section{Si detectors}

Si detectors were not normally considered for use in gamma-ray detection because of Si's low atomic number $(Z)$ and therefore low photopeak efficiency. However, in the specific case of gamma-ray imaging using Compton cameras, not only can Si be used, but it actually presents several advantages compared to Ge or other high-Z materials. First of all, Compton scattering in Si is the predominant interaction versus photoelectric absorption down to the energy of $60 \mathrm{keV}$. This would allow Compton cameras to operate at lower energies than is possible with higher $\mathrm{Z}$ detectors. Also, the distance between gamma-ray interactions would be larger on average in $\mathrm{Si}$, making it easier to reconstruct accurately the direction of incoming gamma rays. In addition, Doppler broadening is smaller in lower $\mathrm{Z}$ materials. ${ }^{20}$ Such a Doppler effect adds to the uncertainty in the reconstructed direction of incoming gamma rays, thereby reducing the image resolution of a Compton camera. Another important advantage of using silicon detectors is that they can be operated at much higher temperatures than Ge detectors, thus simplifying the cooling systems required to operate the detectors.

To achieve good detection efficiency in a Compton camera, silicon detectors can be used as the scatter layer, with a high $\mathrm{Z}$ detector layer as the absorber to fully capture the gamma rays. An all-silicon Compton camera is also feasible using the "3-Compton" reconstruction method. ${ }^{21}$ This method relies on the fact that the measurement of only three Compton scatter events is sufficient to determine the energy and the direction (within a cone surface) of the incident gamma ray photon. Therefore full absorption of the gamma ray is not necessary, and good detection efficiency can be achieved even with low-Z materials such as $\mathrm{Si}$.

Position information of gamma ray interactions in a Si detector can be obtained using the orthogonal-strip configuration. While such detectors fabricated from highresistivity $\mathrm{Si}$ are already commercially available, their thickness is limited to a maximum of $\sim 2 \mathrm{~mm}$. For Compton cameras, the total thickness of Si required to achieve reasonable efficiency is typically on the order of several $\mathrm{cm}$. Thicker detectors are therefore desirable in order to reduce the number of detectors and readout channels. This can be realized using lithium-drifted $\mathrm{Si}(\mathrm{Si}(\mathrm{Li}))$ detectors, which can be made up to $1 \mathrm{~cm}$ thick. Although $\mathrm{Si}(\mathrm{Li})$ detectors have been available for several decades, robust methods to 
fabricate orthogonal-strip contact structures have only recently been developed. ${ }^{22,23}$ In the LBNL process, ${ }^{23}$ we use a contact technology similar to that of the a-Ge contacts for Ge detectors, except that amorphous-Si (a-Si) is employed instead of a-Ge. The a-Si contact replaces the Li contact of the $\mathrm{Si}(\mathrm{Li})$ detector, but, unlike the case of the Ge detectors, it does not work well as a p-type contact. Therefore, B ion implantation is used to form the p-type contact. The B contact replaces the fragile Au surface barrier contact commonly used on $\mathrm{Si}(\mathrm{Li})$ detectors. The B contact has to be formed prior to Li drifting since the high-temperature annealing required to activate the $\mathrm{B}$ would destroy the Li compensation. Segmentation of the B contact by chemical etching is then carried out after Li drifting is completed.

Fig. 5 shows a photograph of a 20 X 20 orthogonal strip Si(Li) detector we have fabricated recently. The strip pitch is $2 \mathrm{~mm}$, with a gap of $0.5 \mathrm{~mm}$ between strips. This detector has a thickness of $3.5 \mathrm{~mm}$. Good energy resolution $(\sim 2 \mathrm{keV} \mathrm{FWHM})$ has been obtained at operating temperature of $\sim 220 \mathrm{~K}$, as shown by the ${ }^{57} \mathrm{Co}$ spectra in Fig. 6 . Thicker detectors can be produced using the same process, with simply longer time required for Li drifting. Efforts to employ such detectors in Compton cameras are ongoing.

\section{CdZnTe detectors}

The use of CdZnTe crystals as gamma ray detectors started around $1991 .^{24}$ $\mathrm{CdZnTe}$ is a high $\mathrm{Z}$, wide bandgap semiconductor. The wide bandgap of CdZnTe allows detectors to operate at room temperature with low leakage currents. In addition, the average $\mathrm{Z}$ of $\mathrm{CdZnTe}$ is significantly higher than that of Ge, which could potentially yield higher photopeak efficiency. These features make CdZnTe extremely attractive as a gamma-ray detector material. Recently, significant progress has been made in growing large single-crystal CdZnTe for detector fabrication. ${ }^{25,26}$ However, present day CdZnTe, like other compound semiconductor detector materials, exhibits poor carrier transport properties. In particular, the hole mobility-lifetime product is very small, at $\sim 5 \mathrm{X} 10^{-5}$

$\mathrm{cm}^{2} / \mathrm{V}$. Electron transport is generally much better, at $\sim 5 \times 10^{-3} \mathrm{~cm}^{2} / \mathrm{V}$, but it is still orders of magnitude worse than that of Ge. Because of the poor carrier transport, conventional planar CdZnTe detectors typically give poor spectral response. This is a result of large variations in detector signals depending on the depth of gamma ray interactions. In order 
for such detectors to achieve energy resolution close to that of $\mathrm{Ge}$, the mobility-lifetime products for electrons and holes in CdZnTe would have to increase by several orders of magnitude. It seems highly unlikely that such large improvements can be achieved in the foreseeable future. Fortunately, alternate techniques have been developed to circumvent some of these material deficiencies.

A significant development was the use of "electron only" charge sensing devices. These devices make use of special detector electrode geometries to improve detector response. One method makes use of the "small pixel effect", which includes detectors with pixellated anodes ${ }^{27}$ or hemispherical geometry ${ }^{28}$. Improved spectral response is achieved because the charge induction property of the detector is modified by the electrode configuration such that events generating charge at different depths in the detector produce signals with less variation compared to simple planar detectors. Best results are obtained by optimizing the electrode geometry in accordance with the carrier transport properties of the material. In most cases, however, the ultimate performance achievable using such devices is fundamentally limited. ${ }^{29}$ Further improvements can be achieved by employing electronic correction techniques in conjunction with pixel detectors. ${ }^{30,31}$ Although very good energy resolution can be obtained with these techniques, the complexity of the electronics, both to readout individual pixels of a detector and to perform the correction, is considerable.

Our group has focused our effort on the development of a different technique called the "coplanar-grid". ${ }^{32,33}$ The coplanar-grid technique utilizes two interdigital coplanar grid electrodes to sense the motion of charge carriers in the detector. A coplanar-grid CdZnTe detector and the readout scheme are depicted in Fig. 7. During operation, a high voltage $\left(-\mathrm{V}_{\mathrm{b}}\right)$ is applied to the cathode to sweep carriers across the detector, and a small voltage $\left(\mathrm{V}_{\mathrm{g}}\right)$ is applied between the two grids to direct the electrons towards one of the grids (collecting grid) and away from the other (non-collecting grid). The signals induced on these two grids by the movement of carriers are subtracted to give a net output signal. This signal can then be processed the same way as that of a conventional detector to perform spectroscopy. This differential signal measurement produces a detector signal that is predominantly determined by the number of electrons reaching the collecting grid. The problem of poor hole collection is then largely eliminated. In addition, by adjusting the relative gain of the two grid signals before 
subtraction, the effects of electron trapping can be nearly perfectly compensated for. ${ }^{29}$ The net result is that the detector signals become independent of the depths of gamma-ray interactions over much of the detector volume, and therefore excellent energy resolution can be obtained.

Fig. 8 shows the very large improvement in spectral response that is achieved using the coplanar-grid technique for a $1 \mathrm{~cm}^{3} \mathrm{CdZnTe}$ detector. Although the energy resolution is still significantly worse than Ge, it is several times better than that of scintillation detectors. With the coplanar-grid technique, it is theoretically possible to achieve resolution close to that of Ge detectors given the typical carrier lifetime already achieved with present CdZnTe materials. The two main factors that are now limiting the energy resolution are material non-uniformity and electronic noise. Progress is being made in understanding these problems and to further improve detector performance. ${ }^{34,35}$

The ability of CdZnTe detectors to operate at room temperature and the simplicity of electronics required to implement the coplanar-grid technique make it possible to build highly compact detection systems. Fig. 9 shows a photograph of a prototype portable detection system we have assembled using a $1 \mathrm{~cm}^{3}$ coplanar-grid CdZnTe detector and a commercial MCA. A much more compact system, containing a $2 \mathrm{~cm}^{3}$ detector, is currently being developed. Despite the relatively small volume of these detectors at the present time, the detection efficiency is quite good due to the high $\mathrm{Z}$ of CdZnTe. As an illustration, Fig. 10 shows a comparison of ${ }^{137} \mathrm{Cs}$ spectra obtained from a $24 \mathrm{~cm}^{3} \mathrm{Ge}$ detector and a $1 \mathrm{~cm}^{3} \mathrm{CdZnTe}$ coplanar-grid detector. The spectra were taken with different source-to-detector distances so absolute efficiency comparison cannot be made, but valid comparisons can be made using peak-to-Compton ratios. The Ge detector has a higher peak-to-Compton ratio as normally defined, but this is mainly due to its much better energy resolution. If the integrated photopeak counts are used to compute an "integrated photopeak"-to-Compton ratio, the CdZnTe detector is actually a factor of two better than the Ge detector. In other words, the $\mathrm{CdZnTe}$ detector is twice as efficient in capturing the full energy of the gamma rays than the Ge detector, even though the Ge detector has 1.5 times the thickness and 24-times the volume.

\section{Conclusions}


After 40 years since the initial development of Ge(Li) detectors, the technology of semiconductor gamma ray detectors continues to advance. The recent development of large segmented $\mathrm{Ge}$ and $\mathrm{Si}$ detectors makes possible the creation of high performance imaging systems. The combination of imaging and high-resolution spectroscopy afforded by these detectors provides a powerful new capability for the detection of gamma-ray sources. In the area of room-temperature detectors, the availability of high quality CdZnTe materials coupled with the development of novel charge sensing techniques has made it possible to produce highly compact detection systems with good energy resolution and efficiency.

\section{Acknowledgment}

This work was supported by the U.S. Department of Energy under Contract No. DE-AC03-76SF00098.

\section{References}

1. A. J. TAVENDALE, IEEE Trans. Nucl. Sci., NS-11 (1964) 191, and references therein.

2. R. D. BAERTSCH, R. N. Hall, IEEE Trans. Nucl. Sci., NS-17 (1970) 235.

3. J. M. MARLER, P. V. HEWKA, IEEE Trans. Nucl. Sci., NS-21 (1974) 287.

4. R. N. HALL, T. J. SOLTYS, IEEE Trans. Nucl. Sci., NS-23 (1976) 88.

5. L. KAUFMAN, W. ROWAN, B. PAYNE, J. HOFFMAN, R. HENCK, W. SCHOEMAEKERS, D. CAMP, IEEE Trans. Nucl. Sci., NS-28 (1981) 117.

6. J. VERPLANCKE, W. SCHOENMAEKERS, Physica Scripta, T32 (1990) 218.

7. P. SANGSINGKEOW, K. D. BERRY, E. J. DUMAS, T. W. RAUDORF AND T. A. UNDERWOOD, presented at the Symposium on Radiation Measurements and Applications, May 21-23, 2002. To be published in Nucl. Instr. Meth.

8. E. E. FENIMORE, T. M. CANNON, Appl. Opt., 17 (1978) 337.

9. G. W. PHILLIPS, Nucl. Instr. Meth., B99 (1995) 674, and references therein.

10. P. N. LUKE, IEEE Trans. Nucl. Sci., 31 (1984) 312. 
11. D. GUTKNECHT, Nucl. Instr. Meth., A288 (1990), 13.

12. K. VETTER, A. KUHNA, M. A. DELEPLANQUE, I. Y. LEE, F. S. STEPHENS, G. J. SCHMID, D. BECKEDAHL, J. J. BLAIR, R. M. CLARK, M. CROMAZ, R. M. DIAMOND, P. FALLON, G. J. LANE, J. E. KAMMERAAD, A. O. MACCHIAVELLI AND C. E. SVENSSON, Nucl. Instr. Meth., A452 (2000) 223.

13. Y. F. YANG, Y. GONO, S. MOTOMURA, S. ENOMOTO, Y. YANO, IEEE Trans. Nucl. Sci., 48 (2001) 656.

14. B. F. PHLIPS, W. N. JOHNSON, R. A. KROEGER, J. D. KURFESS, G. PHILLIPS, E. A. WULF, P. N. LUKE, IEEE Trans. Nucl. Sci., 49 (2002) 597.

15. P. N. LUKE, C. P. CORK, N. W. MADDEN, C. S. ROSSINGTON, M. F. WESELA, IEEE Trans. Nucl. Sci., 39 (1992) 590.

16. M. AMMAN AND P. N. LUKE, Proc. SPIE, 4141 (2000) 144.

17. E. L. HULL, M. T. BURKS, C. P. CORK, W. CRAIG, D. ECKELS, L. FABRIS, A. LAVIETES, P. N. LUKE, N. W. MADDEN, R. H. PEHL AND K. P. ZIOCK, Proc. SPIE, 4507 (2001) 132.

18. M. AMMAN AND P. N. LUKE, Nucl. Instr. Meth., A452 (2000) 155.

19. E. A. WULF, J. AMPE, W. N. JOHNSON, R. A. KROEGER, J. D. KURFESS, B. E. PHLIPS, IEEE Trans. Nucl. Sci., 49 (2002) 1876.

20. D. V. RAO, T. TAKEDA, Y. ITAI, S. M. SELTZER, J. H. HUBBELL, T. ZENIYA, T. AKATSUKA, R. CESAREO, A. BRUNETTI, G. GIGANTE, Proc. SPIE, 4508 (2001) 157.

21. R. A. KROEGER, W. N. JOHNSON, J. D. KURFESS, B. F. PHLIPS, E. A. WULF, IEEE Trans. Nucl. Sci., 49 (2002) 1887.

22. D. PROTIC, T. KRINGS, R. SCHLEICHERT, IEEE Trans. Nucl. Sci., 49 (2002)1993.

23. I. D. HAU, C. TINDALL, P. N. LUKE, presented at the Symposium on Radiation Measurements and Applications, May 21-23, 2002. Nucl. Instr. Meth., in press.

24. J. F. BUTLER, C. L. LINGREN, F. P. DOTY, IEEE Trans. Nucl. Sci., 39 (1992) 605.

25. C. SZELES, S. E. CAMERON, J. O. NDAP, W. C. CHALMERS, IEEE Trans. Nucl. Sci., 49 (2002) 2535.

26. L. LI, F. LU, K. SHAH, M. SQUILLANTE, L. CIRIGNANO, W. YAO, R. W. OLSON, P. LUKE, Y. NEMIROVSKY, A. BURGER, G. WRIGHT, R. B. JAMES. 2001 IEEE Nuclear Science Symposium Conference Record, 4 (2002) 2396. 
27. H. H. BARRETT, J. D. ESKIN, H. B. BARBER, Phys. Rev. Lett., 75 (1995) 156.

28. P. MORTREAU, R. BERNDT, Nucl. Instr. Meth., A 458 (2001) 183.

29. P. N. LUKE, Nucl. Instr. Meth., A 380 (1996) 232.

30. Z. HE, F. ZHANG, G. F. KNOLL, D. K. WEHE, J. BERRY, presented at the Symposium on Radiation Measurements and Applications, May 21-23, 2002. To be published in Nucl. Instr. Meth.

31. L. VERGER, M. BOITEL, M. C. GENTET, R. HAMELIN, C. MESTAIS, F. MONGELLAZ, J. RUSTIQUE, G. SANCHEZ, Nucl. Instr. Meth., A 458 (2001) 297.

32. P. N. LUKE, IEEE Trans. Nucl. Sci., 42 (1995) 207.

33. M. AMMAN AND P. N. LUKE, IEEE Trans. Nucl. Sci., 46 (1999) 205.

34. M. AMMAN, J. S. LEE, P. N. LUKE, J. Appl. Phys., 92 (2002) 3198.

35. P. N. LUKE, J. S. LEE, M. AMMAN, K. M. YU, IEEE Trans. Nucl. Sci., 49 (2002) 1950. 


\section{Figure Captions}

Fig. 1. Maximum volume of high-purity Ge detectors as a function of time.

Fig. 2. Structure of the a-Ge contact.

Fig. 3. A large Ge orthogonal-strip detector fabricated with a-Ge contacts. The detector is $2 \mathrm{~cm}$ thick, with an active area of $9 \mathrm{~cm} \mathrm{X} 9 \mathrm{~cm}$. The active area contains a guard ring electrode and 64 strip electrodes on each side. The strip pitch is $1.25 \mathrm{~mm}$, with a gap of $0.25 \mathrm{~mm}$ between strips.

Fig. 4. Images of radioactive sources taken with a 19 X 19 orthogonal-strip Ge detector. The sources are (from left to right) ${ }^{57} \mathrm{Co},{ }^{241} \mathrm{Am}$, and ${ }^{133} \mathrm{Ba}$. The top left image is from the three sources with no energy selection. The other three images were obtained with energy windows set to the corresponding gamma-ray lines of each of the sources. The histograms at the right and bottom of each image are counts per pixel of sections through the image indicated by the crosshairs.

Fig. 5. A 20 X 20 orthogonal strip $\mathrm{Si}(\mathrm{Li})$ detector. The mirror shows the strip electrodes on the back of the detector. The strip pitch is $2 \mathrm{~mm}$ with a gap of $0.5 \mathrm{~mm}$ between strips. The detector is $3.5 \mathrm{~mm}$ thick.

Fig. 6. ${ }^{57}$ Co spectra obtained from a strip electrode on each side of the detector shown in Fig. 6.

Fig. 7. Photograph of a coplanar-grid CdZnTe gamma-ray detector combined with a schematic diagram of the front-end electronics.

Fig. $8 .{ }^{137}$ Cs spectrum obtained with a $1 \mathrm{~cm}^{3} \mathrm{CdZnTe}$ detector. Top: conventional planar geometry. Bottom: coplanar-grid geometry.

Fig. 9. A prototype portable gamma-ray spectroscopy system utilizing a $1 \mathrm{~cm}^{3} \mathrm{CdZnTe}$ coplanar-grid detector.

Fig. 10. ${ }^{137} \mathrm{Cs}$ spectra obtained with a $24 \mathrm{~cm}^{3}$ Ge planar detector and a $1 \mathrm{~cm}^{3} \mathrm{CdZnTe}$ coplanar-grid detector. 


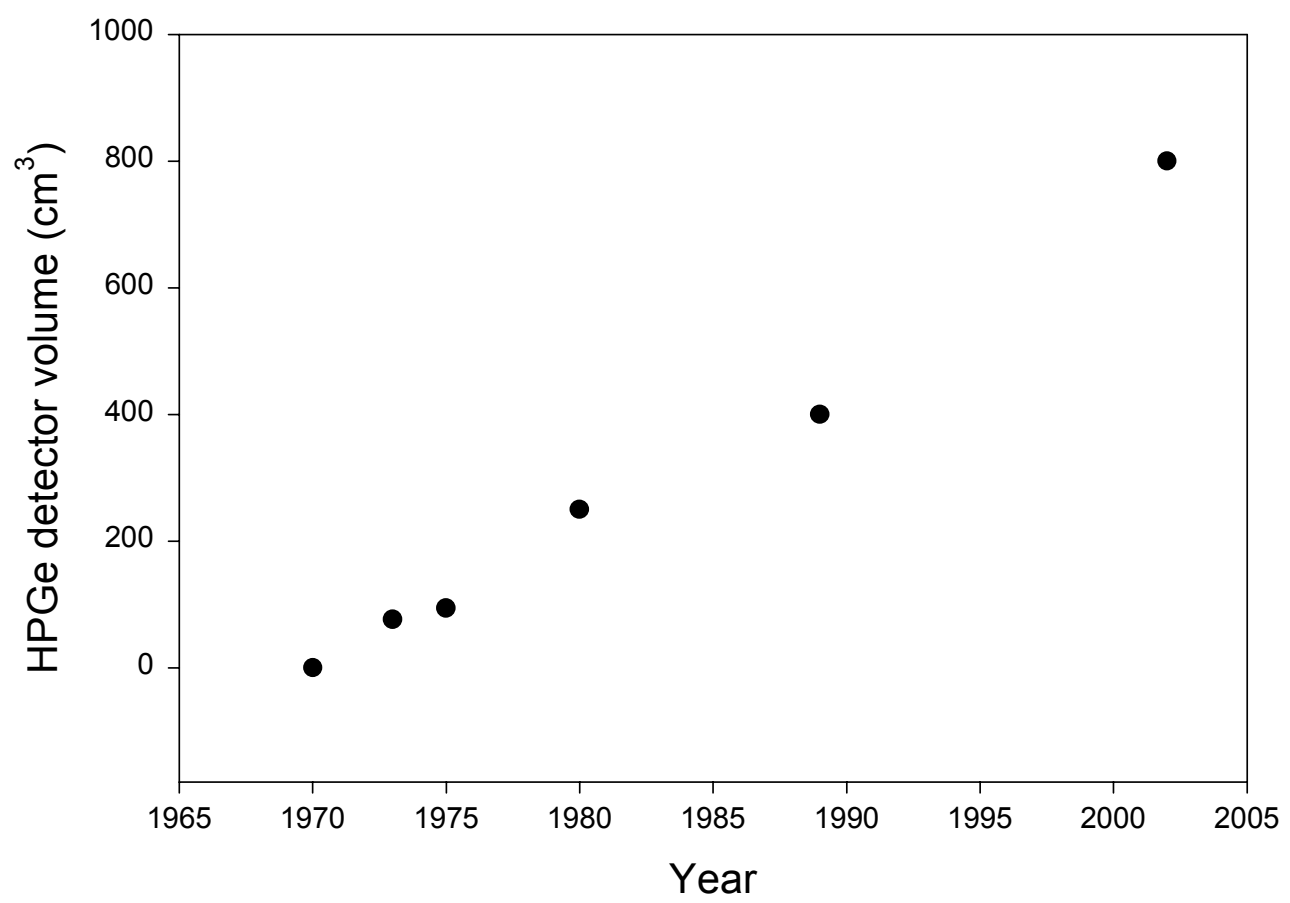

Fig. 1

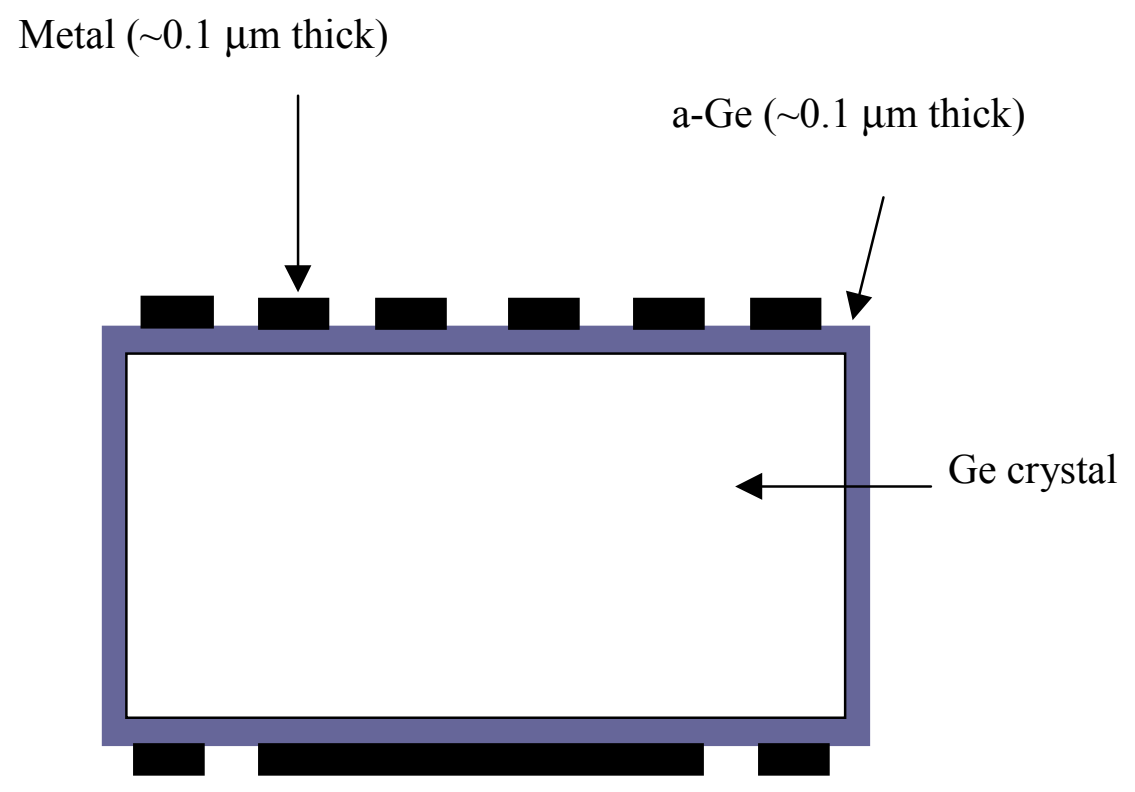

Fig. 2 


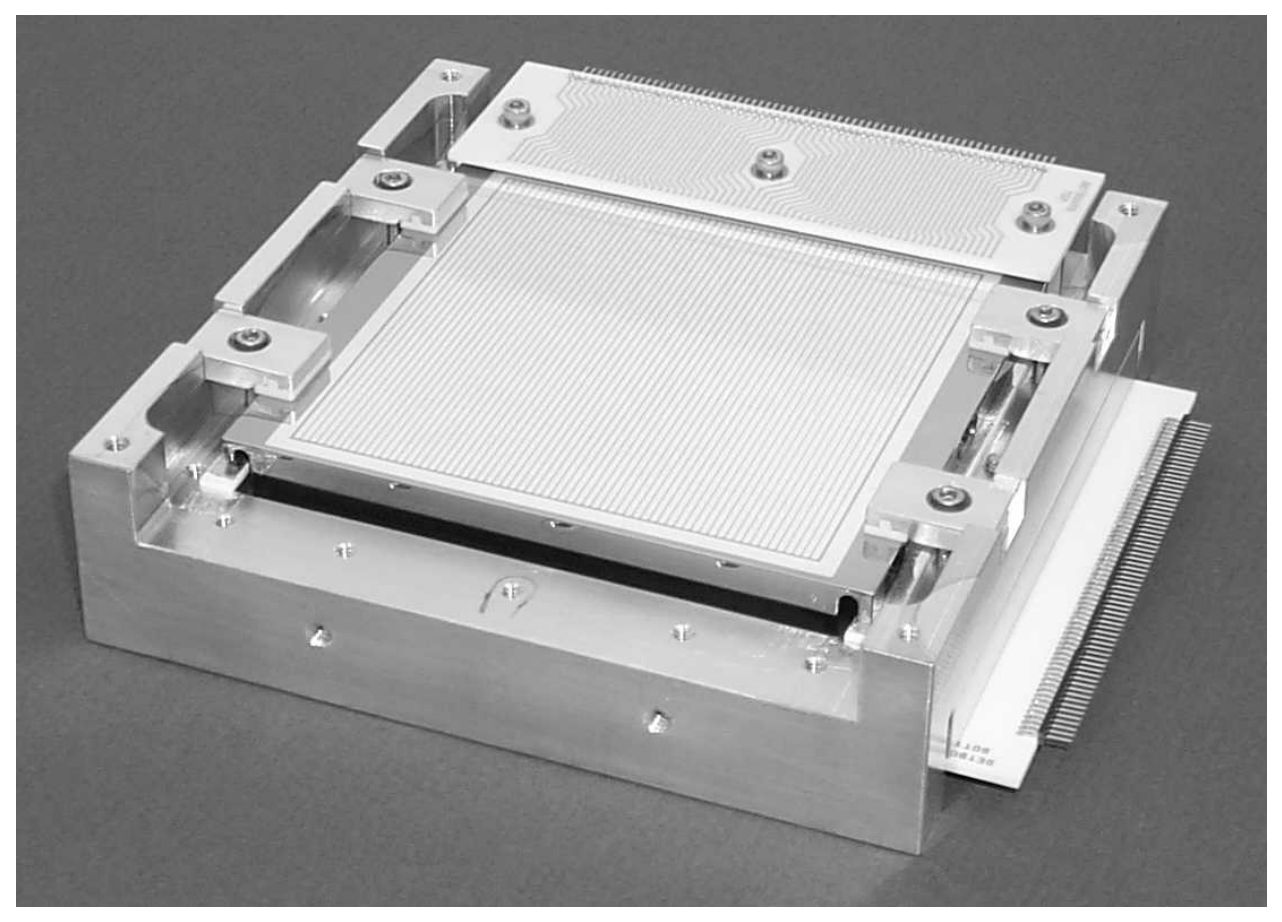

Fig. 3
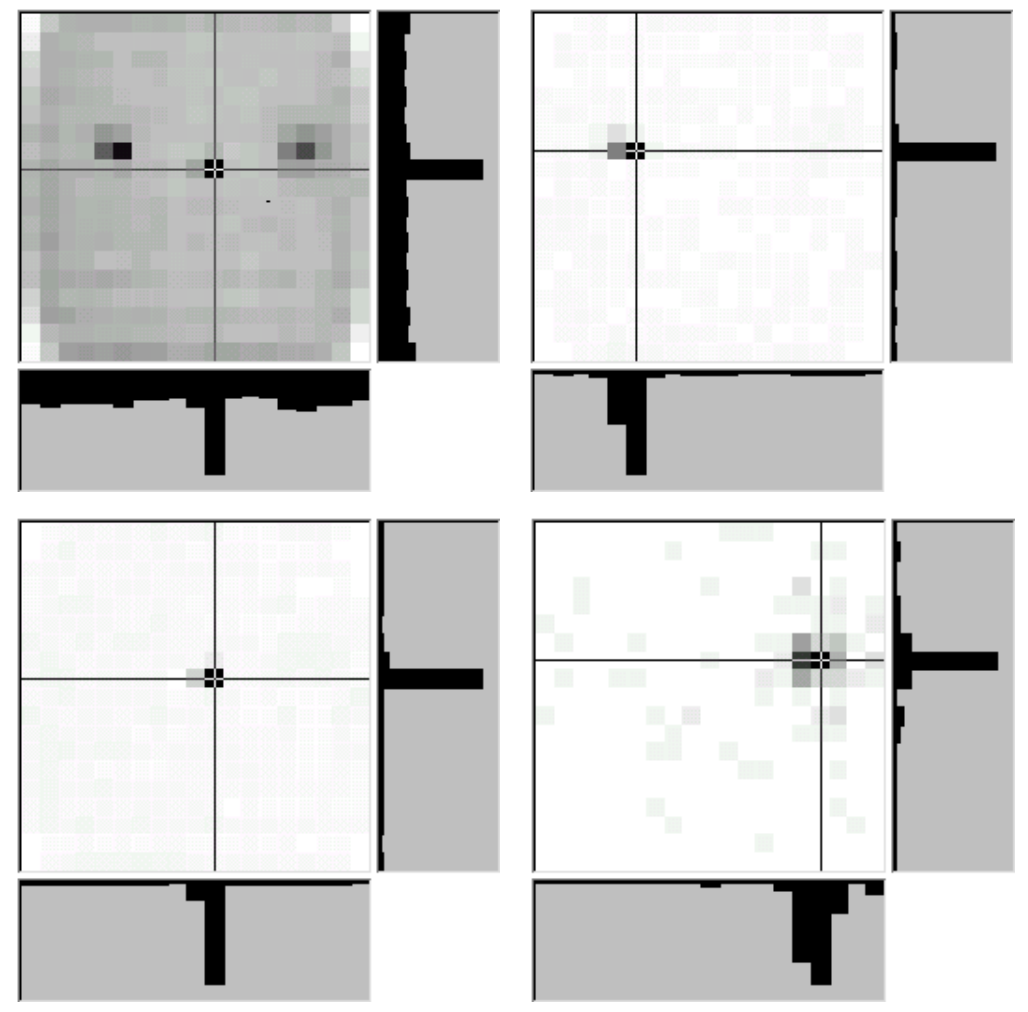

Fig. 4 


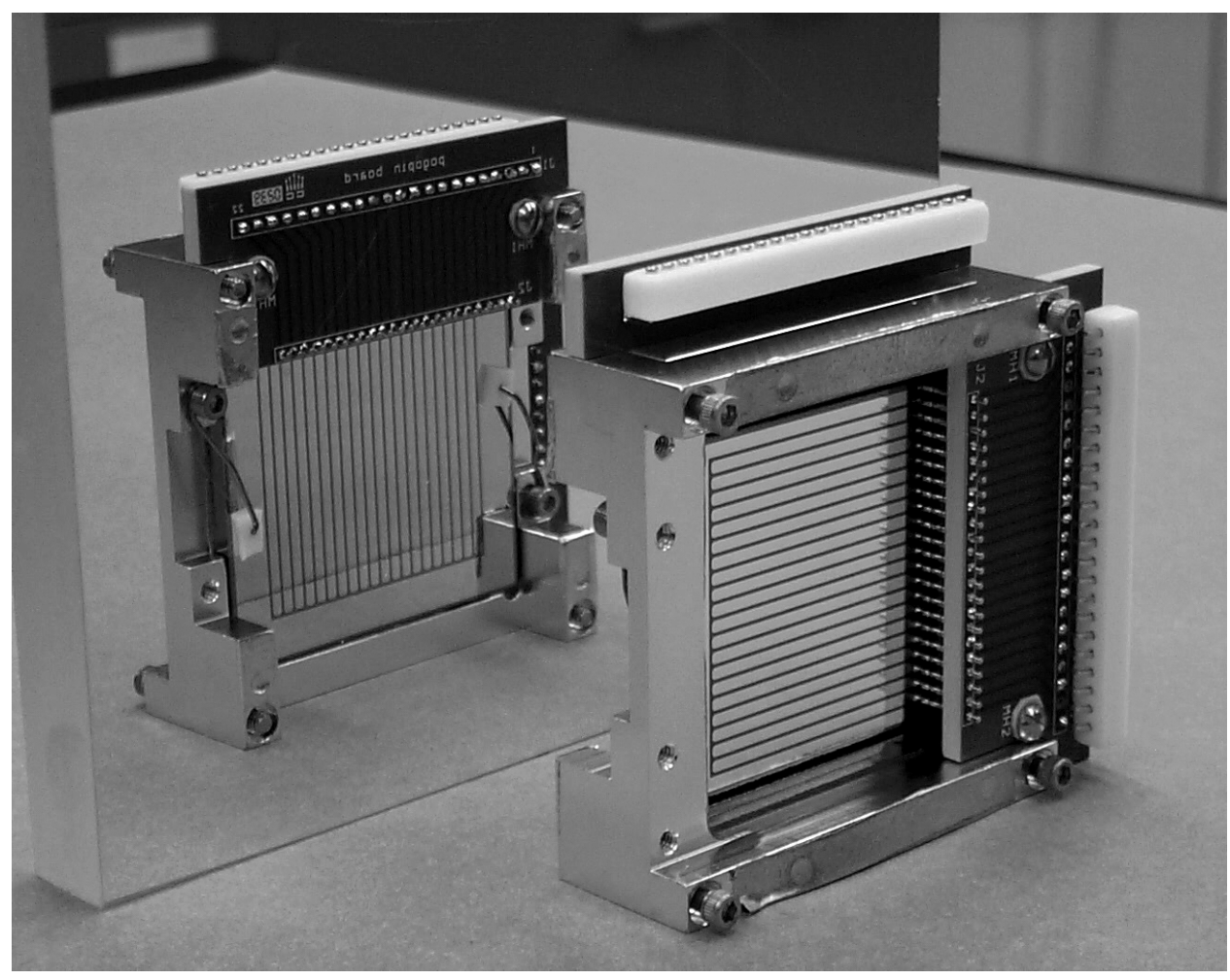

Fig. 5
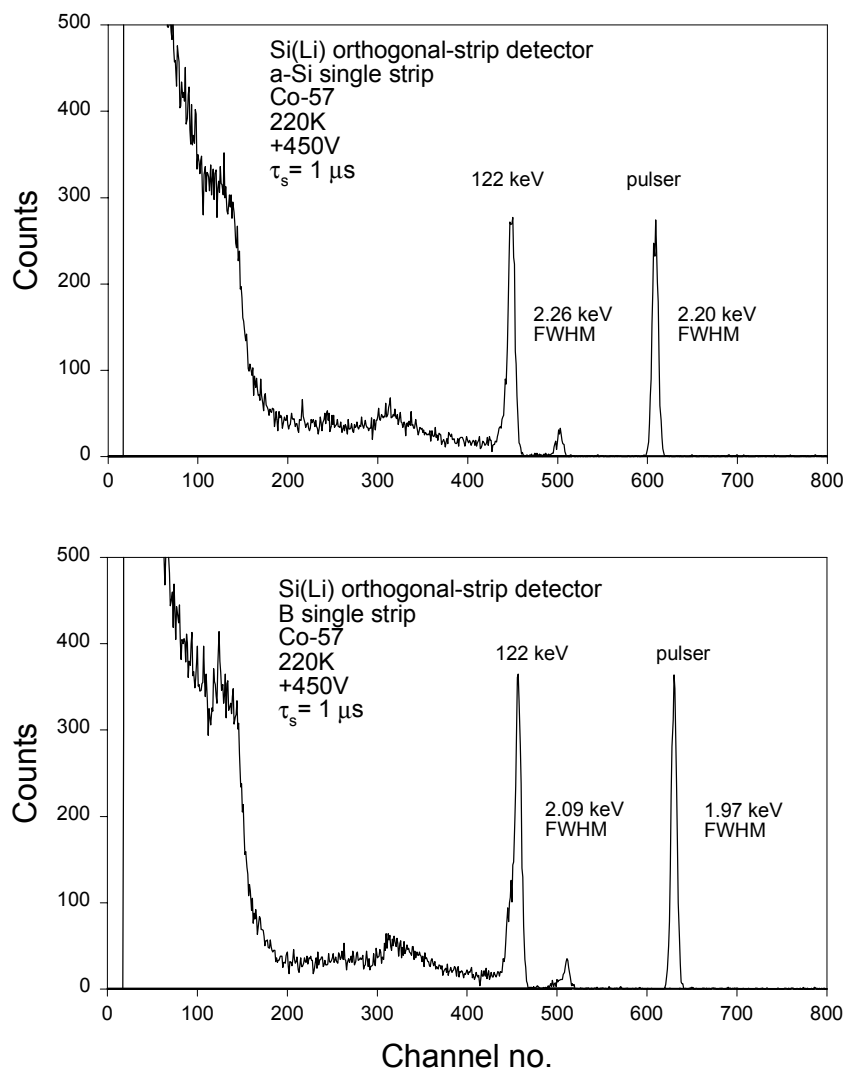

Fig. 6 


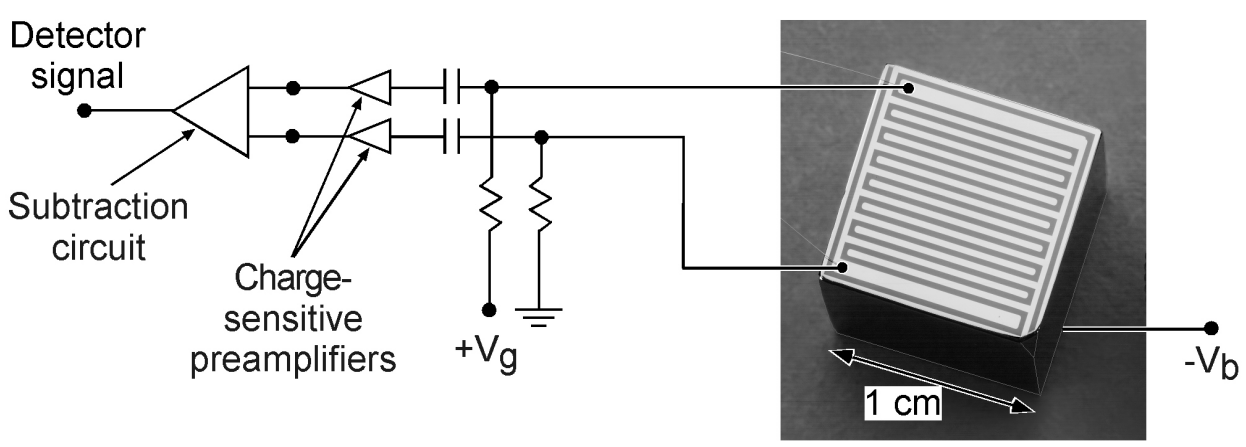

Fig. 7
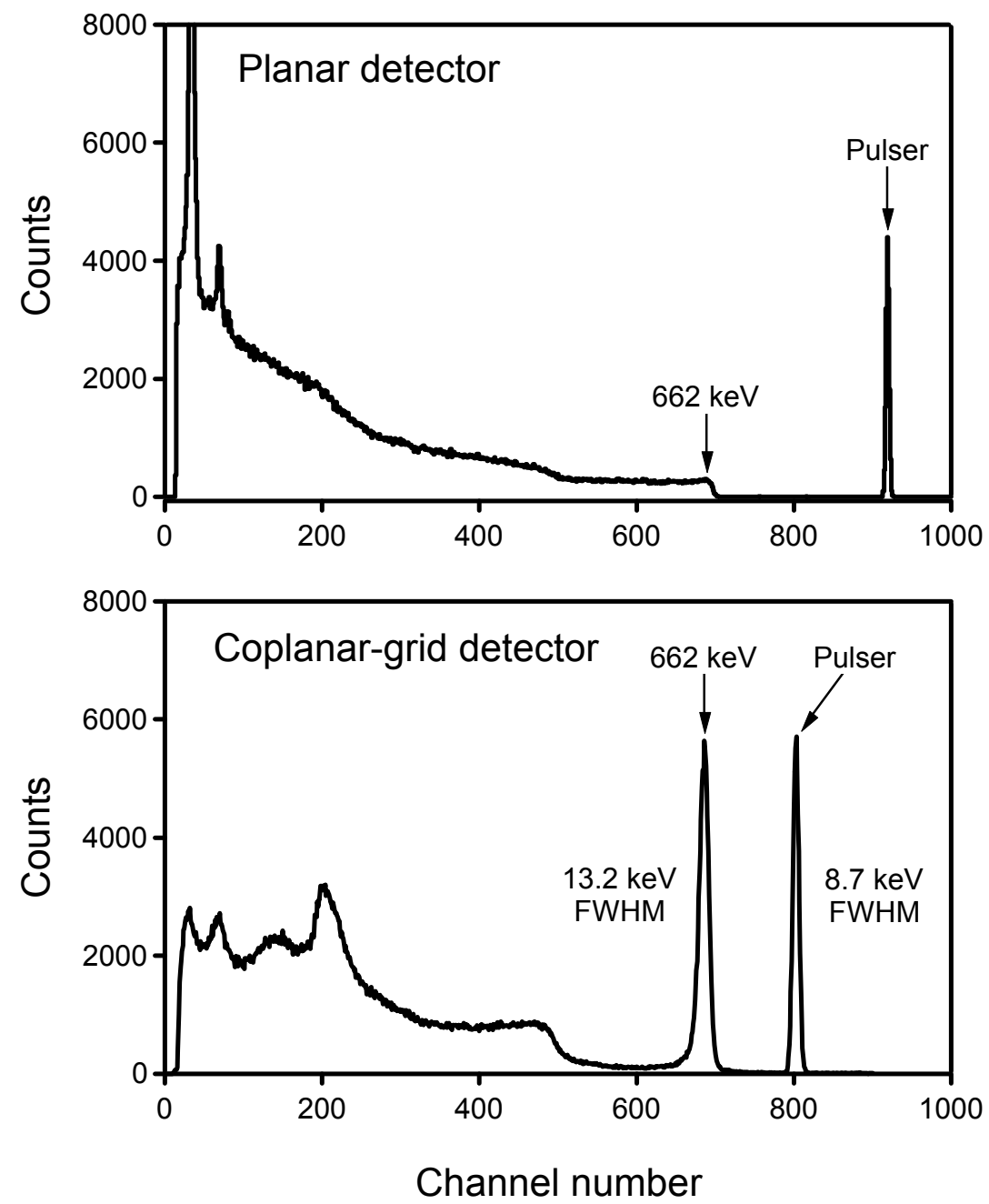

Fig. 8 


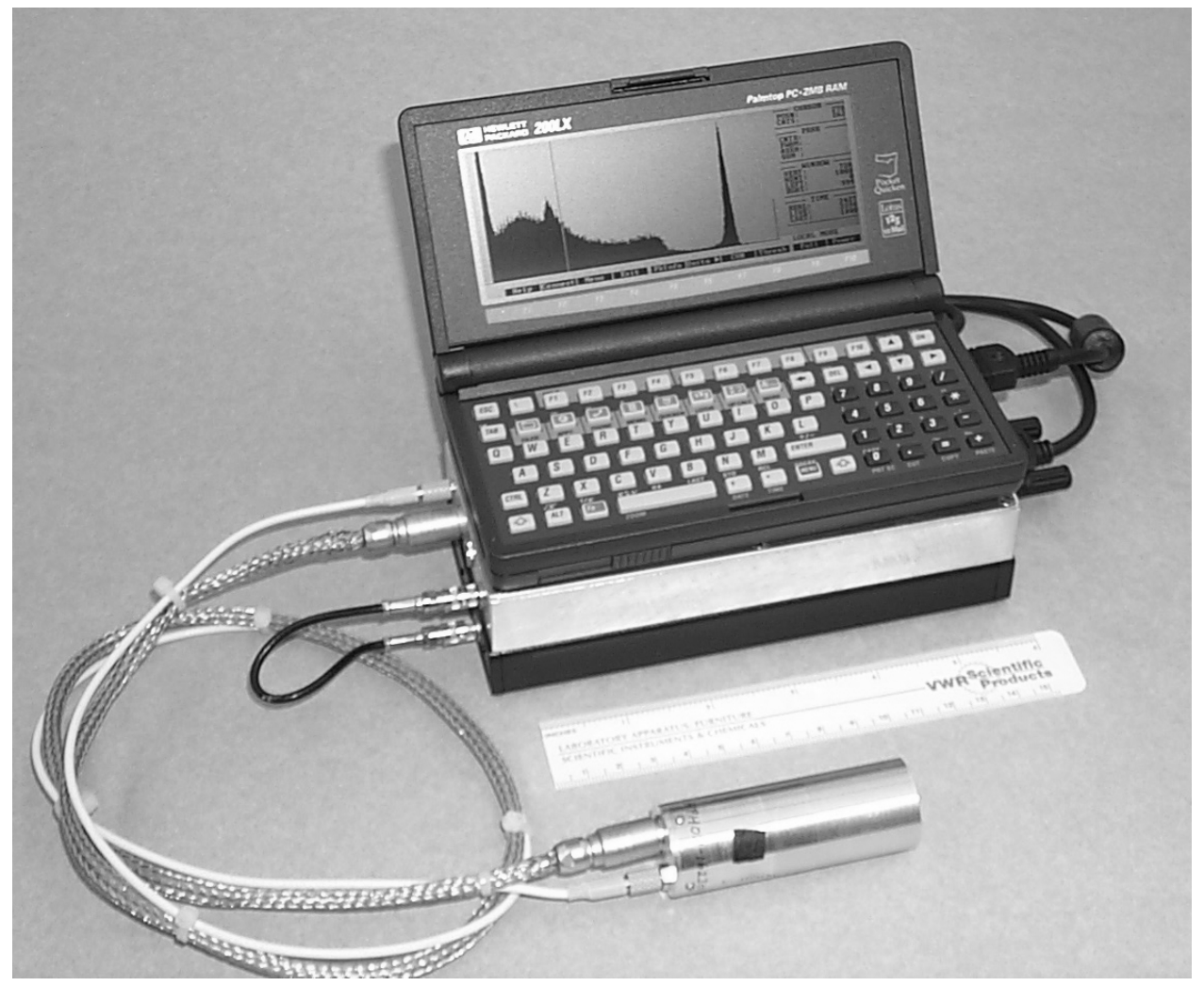

Fig. 9
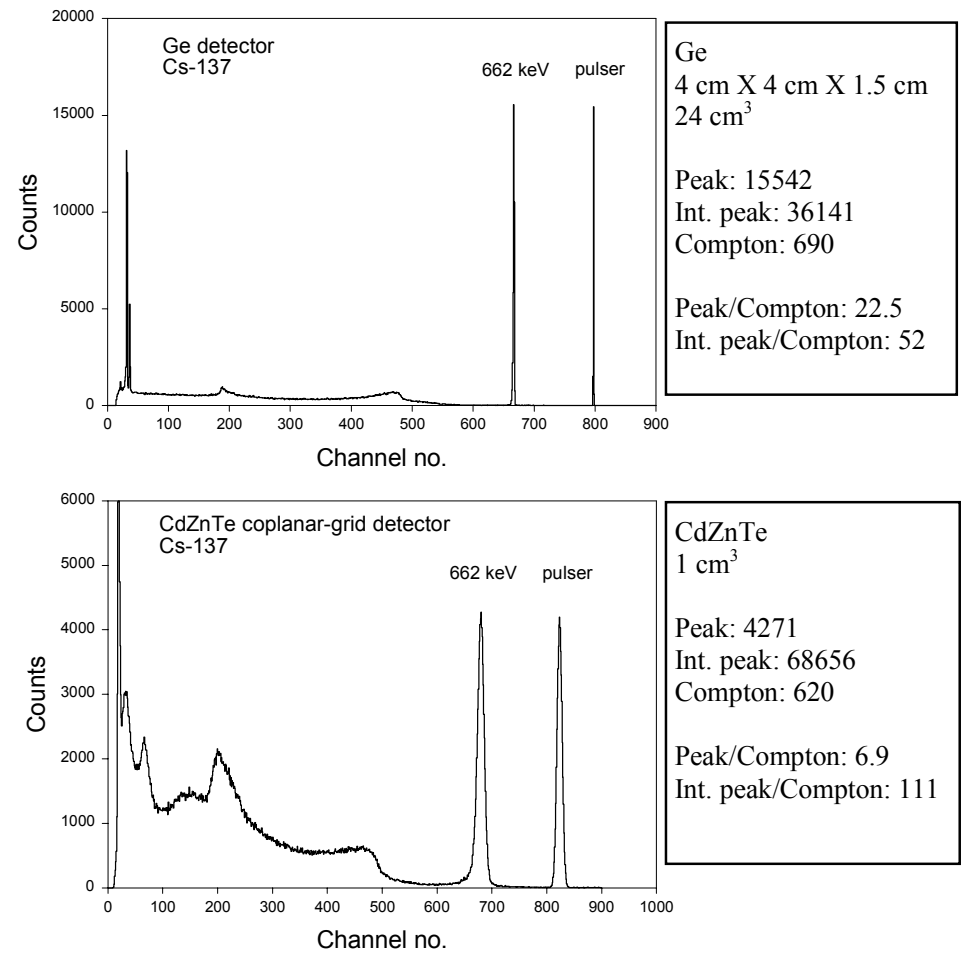

Fig. 10 